\title{
Rupture of aortic aneurysm with right-sided haemothorax
}

\author{
F. Baharloo*, R. Verhelst**, P. Collard*, T. Pieters*
}

\begin{abstract}
Rupture of aortic aneurysm with right-sided haemothorax. F. Baharloo, R. Verhelst, P. Collard, T. Pieters. (C) ERS Journals Ltd 1999.

ABSTRACT: A 62-yr-old male with a history of high blood pressure was admitted for persistent dyspnoea and a right-sided pleural effusion, complicated by a recent episode of shock. There was no history of trauma and the patient denied any thoracic pain. A chest tube was inserted which released nonclotting bloody fluid. A thoracic computed tomographic scan of the chest revealed an aneurysm of the inferior third of the descending thoracic aorta. The patient underwent a successful prosthetic graft replacement.

We emphasize that rupture of aortic aneurysms should be considered in the evaluation of spontaneous haemothorax even if it is right-sided and not associated with pain.

Eur Respir J 1999; 13: 465-467.
\end{abstract}

Rupture of an aneurysm of the thoracic aorta is often a rapidly fatal phenomenon. This report is a case of a non fatal rupture of a descending thoracic aortic aneurysm into the right pleural cavity with some unusual characteristics making a correct diagnosis initially less obvious.

\section{Case report}

A 62-yr-old male was initially admitted to another hospital after he developed a malaise followed by a loss of consciousness. On arrival at the emergency room the patient was in shock. Intravenous administration of fluid allowed a haemodynamic stabilization and the patient regained consciousness. A chest radiograph revealed a massive right pleural effusion. A computed tomographic (CT) scan of the chest confirmed the presence of a right-sided pleural effusion and a mass in the posterior mediastinum was suspected. Thoracentesis of the right pleural cavity yielded a total of $1.5 \mathrm{~L}$ of bloody fluid. A diagnostic thoracoscopy was planned but the patient left the hospital for home 7 days after admission.

Three days later the patient presented to our clinic, complaining of persistent dyspnoea. He had a history of neglected high blood pressure. He had smoked the equivalent of 35 pack-yrs and had stopped smoking 25 yrs previously. He complained of dyspnoea grade III and a mild dry cough but denied having experienced any thoracic pain or tenderness. On physical examination the patient was pale. His blood pressure was $210 / 120 \mathrm{mmHg}$ and cardiac frequency 90 beats $\cdot \min ^{-1}$. Breath sounds were decreased at the right lung base with dullness at percussion. Peripheral pulses were palpated. A chest radiograph revealed a massive right-sided pleural effusion and showed an abnormal convex opacity superposing the right hilum (fig. 1). Hae-
Units of *Pneumology and **Cardiovascular and Thoracic Surgery, Cliniques Universitaires Saint-Luc, Brussels, Belgium.

Correspondence: T. Pieters

Service de Pneumologie

Cliniques Universitaires Saint-Luc

Avenue Hippocrate 10

B-1200 Brussels

Belgium

Fax: 3227643703

Keywords: Spontaneous haemothorax thoracic aortic aneurysm

Received: December 291997

Accepted after revision May 231998

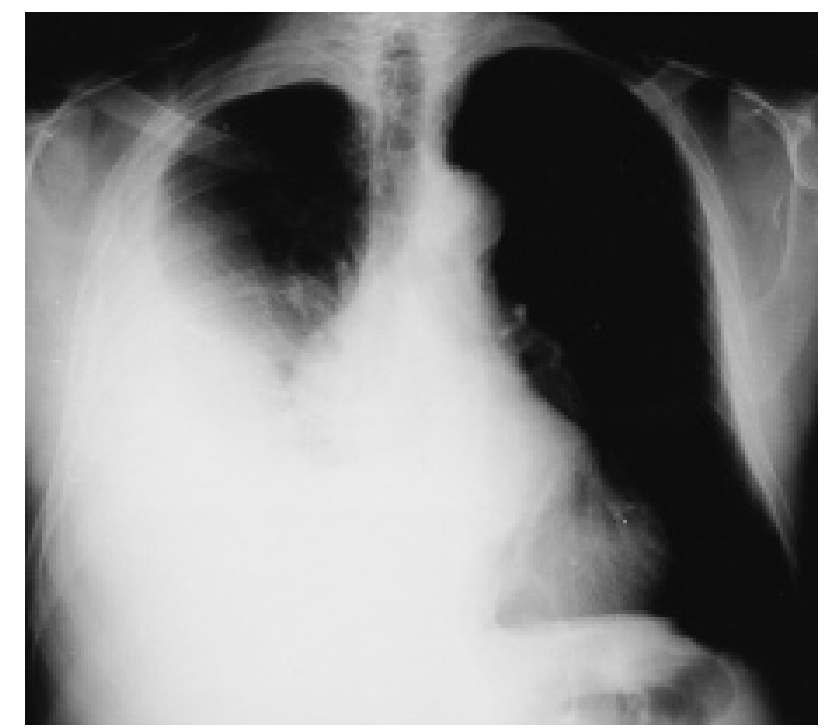

Fig. 1. - Chest radiograph showing a right pleural effusion with an abnormal convex opacity of the level of the right hilum. The computed tomographic scan identified this as a fluid collection in the mediastinum (haemomediastinum) surrounding the ascending aorta.

moglobin was $5.64 \mathrm{mmol} \cdot \mathrm{L}^{-1}$, the haematocrit was 0.28 and the platelet count was $557 \times 10^{9} \cdot \mathrm{L}^{-1}$. Clotting parameters were normal.

A chest tube was inserted yielding $1 \mathrm{~L}$ of nonclotting bloody fluid. A further $2 \mathrm{~L}$ was eliminated during the following $24 \mathrm{~h}$. The complete blood count and chemistry of the pleural fluid were as follows: haematocrit 0.23 , white blood cell count $4 \times 10^{9}$ cells $\cdot \mathrm{L}^{-1}$ with $75 \%$ neutrophils, glucose $3.33 \mathrm{mmol} \cdot \mathrm{L}^{-1}$, protein $70 \mathrm{~g} \cdot \mathrm{L}^{-1}$, lactate dehydrogenase $5,530 \mathrm{IU} \cdot \mathrm{L}^{-1}$ and $\mathrm{pH} 7.2$. 
a)

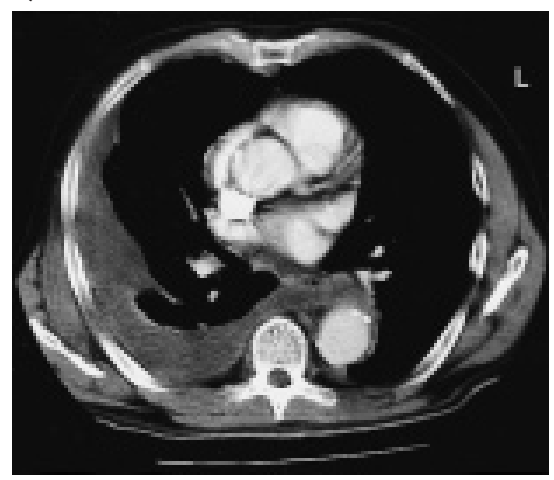

b)

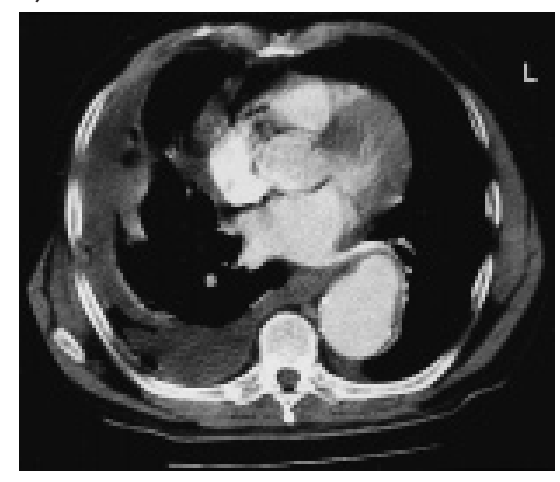

c)

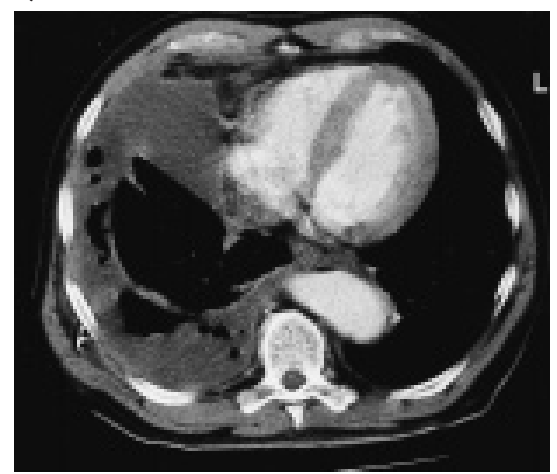

Fig. 2. - Computed tomographic scan sections of the thorax showing: a) a right-sided pleural fluid collection with a normal-sized aorta with three wall calcifications; b) the aortic aneurysm with the aorta bulging obliquely to the left and a mixed air-fluid collection; and c) the aortic wall bulging obliquely to the right, the air-fluid collection and the drain in the muscular tissues.

A CT scan of the thorax revealed an aneurysm of the descending thoracic aorta of $9.7 \mathrm{~cm}$ in length and $6.6 \mathrm{~cm}$ in diameter (fig. 2). Four days after admission a left thoracotomy was performed under general anaesthesia with selective intubation by a double-lumen tube. Multilevel somatosensory evoked potentials (SEP) were obtained by bilateral posterior tibial nerve stimulation with peripheral nerve, lumbar spinal, brainstem and cortical recordings to detect eventual spinal cord ischaemia during the clamping time [1]. No blood was found in the left pleural cavity. A left-to-left atriofemoral shunt using a Biomedicus pump was installed to avoid haemodynamic instability due to a sudden increase in afterload and a risk of ischaemia during clamping. A severely inflammatory fusiform aneurysm of the descending thoracic aorta distal to the left subclavian artery and extending to the diaphragm was confirmed, with inflammatory exudate present in the left pleural cavity. After clamping and opening of the aneurysm, a 2.5-cm-wide circular rupture covered by a fresh clot was identified on its right wall (fig. 3). A gelatincoated dacron graft (Vascutek-Gelseal, Sulzer Medica, Inchinnam, Renfrewshire, UK) was interposed between the proximal and distal aorta by end-to-end anastomosis. During the distal suturing, signs of spinal cord ischaemia were detected by SEP monitoring. Two intercostal arteries were, therefore, rapidly reperfused by oblique anastomosis to the distal aorta.

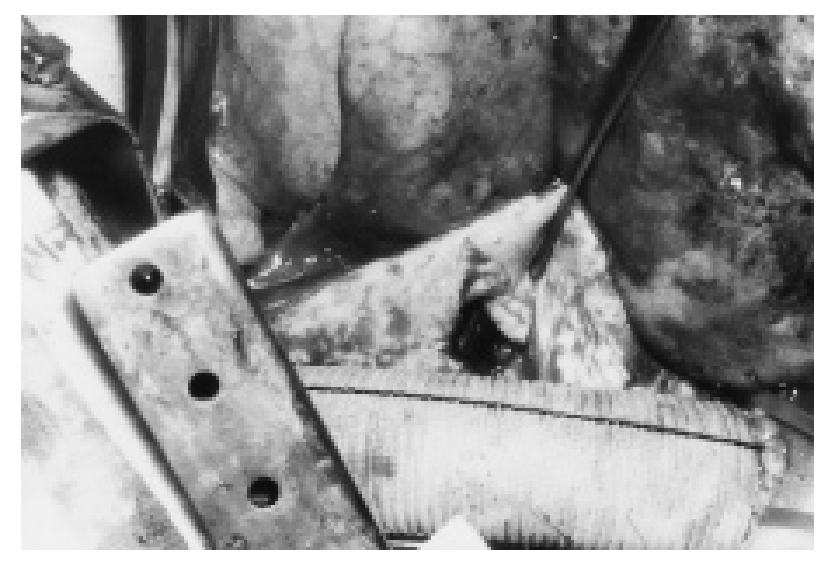

Fig. 3. - Peroperative view of the aneurysm opening. A $2.5-\mathrm{cm}$-wide circular hole is visible on its right wall. The prosthetic graft is in place.
The immediate postoperative period was uneventful. However, a persistent chylothorax finally required a second thoracotomy to repair a damaged ectopic thoracic duct. The patient was discharged from hospital 3 weeks after admission. At 12 months follow-up he had made an excellent recovery.

\section{Discussion}

Haemothorax is defined as pleural effusion with a haematocrit $\geq 50 \%$ that of blood [2]. Pleural fluid imparts a red colour indistinguishable from blood at a haematocrit $\geq 5 \%$. It is, therefore, always important to measure the haematocrit of any haemorrhagic pleural effusion. Care should be taken as secondary dilution of a haemothorax can reduce its haematocrit to well below 50\% that of blood within a few days, making it appear as a haemorrhagic exudate [3]. Neoplasm, tuberculosis and pulmonary infarction should be considered in priority in the differential diagnosis of a blood-tinged pleural effusion. A true haemothorax however, corresponds to a vascular disruption resulting in bleeding into the pleural cavity.

The majority of haemothoraces are due to blunt or penetrating trauma or are iatrogenic in origin, whereas nontraumatic or spontaneous haemothorax is a rare occurrence. Various possible aetiologies have been described in the literature, mainly as case reports. Two of the most frequent causes are haemopneumothorax and anticoagulation therapy [3]. Other causes include primary coagulopathies, systemic or pulmonary vascular erosion by a tumour or exostoses, pulmonary arteriovenous malformations, endometriosis and systemic lupus erythematosis [3]. The source of bleeding can also be subdiaphragmatic [3]. The importance of a thorough examination of the abdomen should, therefore, not be underestimated.

Rupture of the thoracic aorta into the pleural cavity, principally due to aortic dissection, has been regularly described in the literature. The ascending thoracic aorta bleeds mainly into the pericardium. For obvious anatomical reasons, the ruptured descending thoracic aorta bleeds preferentially into the left pleural cavity. We know of three cases of right-sided bleeding described in the surgical literature [4-6]. They all had an acute presentation and were surgically treated with success. In the case of the present 
patient, a fusiform aneurysm of the descending thoracic aorta ruptured along the right side of its wall, bleeding into the right pleural cavity and causing haemorrhagic shock. Luckily, the formation of a clot at the site of the tear prevented fatal exsanguination. The mediastinal structures facing the vessel wall certainly played a part in hampering continuous bleeding.

This case shows how the diagnosis of a rupturing thoracic aorta can easily be overlooked because of an atypical presentation. Given the severity of its prognosis, even if the haemothorax is on the right side, a thoracic aortic rupture should not be excluded. The absence of thoracic pain also does not exclude this diagnosis.

\section{References}

1. Guérit JM, Verhelst R, Rubay J, Khoury G, Matta A, Dion R. Multilevel somatosensory evoked potentials (SEPs) for spinal cord monitoring in descending thoracic and thoraco-abdominal aortic surgery. Eur J Cardiothorac Surg 1996; 10: 93-104.

2. Light RW. Pleural Diseases, 2nd Edn. Philadelphia, PA, Lea and Febinger, 1990; pp. 263-267.

3. Martinez FJ, Villanueva AG, Pickering R, Becker FS, Smith DR. Spontaneous hemothorax, report of six cases and review of the literature. Medicine (Baltimore) 1992; 71: 354-368.

4. Hata T, Namba H, Tsushima Y, et al. A successful surgical case of thoracoabdominal aortic aneurysm ruptured into the right pleural cavity. Kyabu-Geka 1992; 45: 557560 .

5. Katagiri M, Takahashi M. Right hemothorax: an unusual presentation of ruptured aortic dissection. $J$ Cardiovasc Surg 1991; 32: 135-136.

6. van-der-Vliet JA, Heijstraten FM, van-Roye SF, Buskens FG. Spontaneous right haemothorax secondary to aortic rupture. Eur J Vasc Surg 1994; 8: 634-638. 